\title{
Bleomycin/Dacarbazine/Doxorubicin/Lomustine Regimen
}

National Cancer Institute

\section{Source}

National Cancer Institute. Bleomycin/Dacarbazine/Doxorubicin/Lomustine Regimen. NCI

Thesaurus. Code C161917.

A chemotherapy regimen consisting of bleomycin, dacarbazine, doxorubicin and lomustine. 